\title{
Interferometer design of the KAGRA gravitational wave detector
}

\author{
Yoichi Aso, ${ }^{1, *}$ Yuta Michimura, ${ }^{1, \dagger}$ Kentaro Somiya, ${ }^{2,}$ Masaki Ando, ${ }^{1,3}$ Osamu Miyakawa, ${ }^{4}$ \\ Takanori Sekiguchi, ${ }^{4}$ Daisuke Tatsumi, ${ }^{3}$ and Hiroaki Yamamoto ${ }^{5}$
}

(The KAGRA Collaboration)

\author{
${ }^{1}$ Department of Physics, University of Tokyo, 7-3-1 Hongo, Bunkyo-ku, Tokyo 113-0033, Japan \\ ${ }^{2}$ Department of Physics, Tokyo Institute of Technology, 2-12-1, Ookayama, Meguro-ku, Tokyo 152-8550, Japan \\ ${ }^{3}$ National Astronomical Observatory of Japan, 2-21-1 Osawa, Mitaka, Tokyo 181-8588, Japan \\ ${ }^{4}$ Institute for Cosmic Ray Research, University of Tokyo, 5-1-5 Kashiwa-no-Ha, Kashiwa City, Chiba 277-8582, Japan \\ ${ }^{5}$ LIGO Laboratory, California Institute of Technology, Pasadena, California 91125, USA
}

(Received 16 June 2013; published 20 August 2013)

\begin{abstract}
KAGRA is a cryogenic interferometric gravitational-wave detector being constructed at the underground site of Kamioka mine in Gifu prefecture, Japan. We performed an optimization of the interferomter design, to achieve the best sensitivity and a stable operation, with boundary conditions of classical noises and under various practical constraints, such as the size of the tunnel or the mirror cooling capacity. Length and alignment sensing schemes for the robust control of the interferometer are developed. In this paper, we describe the detailed design of the KAGRA interferometer as well as the reasoning behind design choices.
\end{abstract}

DOI: 10.1103/PhysRevD.88.043007

PACS numbers: 95.55.Ym, 42.60.Da

\section{INTRODUCTION}

Direct detection of gravitational waves from astronomical sources will not only be a powerful way to test gravity theories under strong gravitational fields, but also an intrinsically new way to observe the Universe [1]. Such observations will provide us with unique information not available with conventional astronomical observations using electromagnetic waves. Currently, one of the most promising ways to detect gravitational waves is to use large laser interferometers. Several large-scale interferometric gravitational-wave detectors were built and successfully operated to prove the feasibility of such detectors [2]. However, those first generation detectors were still not sensitive enough to detect gravitational waves unless there is an extremely lucky event, such as a nearby neutron star merger. There are several next-generation interferometric gravitational-wave detectors being built around the world [2]. These detectors generally aim at improving the sensitivity by ten-fold from the first-generation detectors to make regular detection a reality.

KAGRA [3] is a Japanese next-generation gravitationalwave detector, now under construction at an underground site in the currently disused Kamioka-mine, in Gifu prefecture, Japan. KAGRA has two outstanding features: cryogenic mirrors made of mono-crystalline sapphire to reduce thermal noises and the seismically quiet and stable environment of an underground site. The detector will be

\footnotetext{
*aso@granite.phys.s.u-tokyo.ac.jp

michimura@granite.phys.s.u-tokyo.ac.jp

\$somiya@phys.titech.ac.jp
}

located inside a large mountain more than $200 \mathrm{~m}$ deep into the mountain body from the surface. The construction of KAGRA started in 2010 and the operation of the detector in its full configuration is planned to begin in 2017.

The development of KAGRA is performed in two phases. The initial KAGRA, or iKAGRA, is the first phase of the operation with a simple Fabry-Perot Michelson interferometer configuration. The main purpose of iKAGRA is to quickly identify facility-related or any other problems at an early stage of construction, thus allowing more time to address these potential issues. The final configuration of KAGRA is called the baseline KAGRA, or bKAGRA. In this paper, we focus on the bKAGRA interferometer and explain its design, the reasoning behind the parameter choices, and so on.

The paper is structured as follows. First, we give a brief overview of the KAGRA interferometer configuration and set the terminology for the later discussion (Sec. II). Then, we briefly review the noise sources of KAGRA with nonquantum origins, namely, seismic noise and thermal noises (Sec. III). We treat these noises as boundary conditions for optimizing the quantum noises (shot noise and radiationpressure noise) in Sec. IV. This process basically determines the reflectivities of the interferometer mirrors. Then we proceed to consider how to control these mirrors and lock the interferometer at the optimal operation point in Sec. V. This boils down to selecting the macroscopic lengths of the recycling cavities and the Michelson asymmetry to realize the optimal resonant conditions for the radio frequency (RF) sidebands used to extract error signals for the interferometer control. In Sec. VI, we consider the spatial-mode properties of the interferometer, 
especially in terms of the ability to reject unwanted higherorder modes. This determines the radii of curvature (ROC) of the mirrors. In Sec. VII, we examine whether reasonable alignment information of the interferometer can be extracted with the selected interferometer parameters. Finally, we give conclusions in Sec. VIII.

\section{OVERVIEW OF THE KAGRA INTERFEROMETER AND TERMINOLOGY}

Before going into the details of the interferometer design, we first give a brief overview of the interferometer configuration of KAGRA and explain the terminology used throughout the rest of this paper.

\section{A. Interferometer configuration}

The schematic view of the KAGRA interferometer is shown in Fig. 1. The laser beam of $1064 \mathrm{~nm}$ wavelength is first passed through a three-mirror optical cavity called a mode cleaner (MC) to clean the spatial mode of the incident beam. After the MC is a main interferometer, which consists of four cryogenic mirrors and seven auxiliary mirrors at room temperature. We have two $3 \mathrm{~km}-$ long Fabry-Perot cavities, called arm cavities, formed by input test masses (ITMs) and end test masses (ETMs). These test masses are cooled down to around $20 \mathrm{~K}$ to reduce thermal noises. The two arm cavities are combined by a beam splitter (BS) and the interference condition on the BS is held such that all the light comes back in the direction of a mirror called PR3. A power-recycling mirror (PRM) and the two ITMs form a power-recycling cavity (PRC). Similarly, a signal-recycling mirror (SRM) forms a signal-recycling cavity (SRC) together with the ITMs. This interferometer configuration with two recycling cavities is called dual-recycling. In particular, we keep the

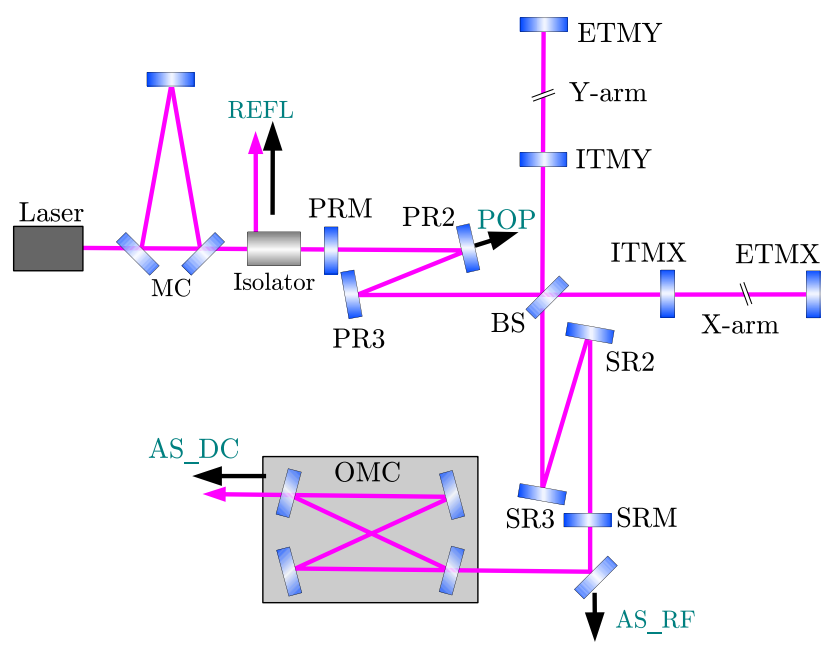

FIG. 1 (color online). Schematic of the KAGRA interferometer. Names of the mirrors as well as the signal detection ports are shown.
TABLE I. Length degrees of freedom of the KAGRA interferometer.

\begin{tabular}{lr}
\hline \hline DARM & Differential length change of the arm cavities. \\
CARM & Common length change of the arm cavities. \\
MICH & Michelson degree of freedom. \\
PRCL & Power-recycling cavity length. \\
SRCL & Signal-recycling cavity length. \\
\hline \hline
\end{tabular}

SRC length to be resonant for the carrier light, which is called the resonant sideband extraction (RSE) scheme.

The PRC and the SRC are folded in Z-shapes by two additional mirrors each for improving the spatial mode stability, as explained in Sec. VI. Downstream of the SRM, there is an output mode-cleaner (OMC) used to remove unwanted higher-order spatial modes from the output beam.

\section{B. Length degrees of freedom}

From the point of view of interference and resonance of the light, there are five length degrees of freedom (d.o.f.) in our interferometer. The names of the d.o.f. are summarized in Table I. All the d.o.f. are represented as linear combinations of the motions of the mirrors. The most important d.o.f. is the differential length change of the arm cavities, called DARM. It contains gravitational-wave information. CARM is the common change of the arm-cavity lengths. $\mathrm{MICH}$, which is short for Michelson, is the differential change of the distances between the BS and the two ITMs. PRCL and SRCL are the lengths of the PRC and the SRC, respectively. Since DARM is the most important d.o.f., the other four d.o.f. are often called auxiliary d.o.f.

\section{Detection ports}

Laser beams coming out of the interferometer are detected at various places for extracting the interferometer information. The names of these detection ports are also given in Fig. 1. The reflection port (REFL) is located at the reflection output of a Faraday isolator, which reflects the light coming back from the interferometer. The light coming out of the SRC is led to two antisymmetric (AS) ports. The beam picked off before the OMC goes to the AS_RF port. The transmission of the OMC is called the AS_DC port. The POP (Pick-Off-in-the-PRC) port is the transmission of the PR2 mirror.

\section{NON-QUANTUM NOISES OF KAGRA}

In this section and the next section, we determine the target sensitivities of KAGRA. As is explained in Sec. IV, we operate the detector with two different states of SRC detuning. Therefore, there are two target sensitivity curves, corresponding to the broad-band RSE (BRSE) configuration and the detuned RSE (DRSE) configuration.

Figure 2 shows the estimated noises of the KAGRA detector. The sensitivity is mostly limited by quantum 

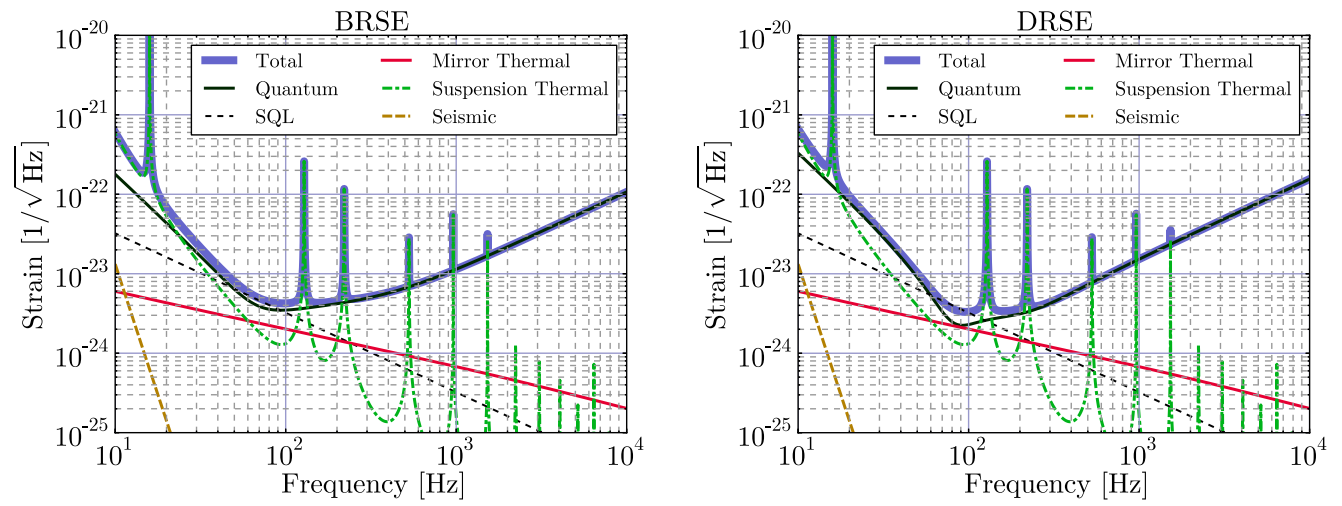

FIG. 2 (color online). Estimated noises of KAGRA. The total noise is the quadratic sum of all the noises.

noises. Suspension thermal noise contributes to the total noise at low frequencies (below $30 \mathrm{~Hz}$ ). In this section we first give an overview of the non-quantum noises. A detailed discussion of these noises are given in Ref. [4].

\section{A. Seismic noise}

Each sapphire test mass is suspended under a complex seismic attenuation system (SAS) that combines a short and sturdy inverted pendulum with a series of suspension stages with geometric antispring (GAS) filters [4]. In order to cool down the mirrors, heat links made of pure aluminum wires are attached to the penultimate and upper stages of the suspension system. The auxiliary mirrors are suspended by simpler suspension systems.

In general the seismic motion of the KAGRA site is very quiet, about 100 times lower than that of the TAMA site in Tokyo [5]. However, the seismic activity depends on season and weather. In order to estimate the seismic noise of the interferometer mirrors, we use the simulated transfer functions of the above-mentioned suspension systems and the measured ground-vibration spectrum of a stormy day in the Kamioka mine, which is a worst-case scenario.

\section{B. Thermal noises}

\section{Heat extraction capacity}

In order to extract heat from the sapphire test masses, the mirrors are suspended by sapphire wires, which have a high thermal conductivity at low temperatures. Then the heat is transferred to the cold heads of the cryocoolers by pure aluminum wires connected to the upper stages of the mirror suspension systems [4].

In the current design, the expected heat absorption by an ITM from the incident laser beam is about $1.2 \mathrm{~W}$ [4]. The diameter of the sapphire wires is determined to be $1.6 \mathrm{~mm}$, so that this heat can be extracted without increasing the mirror temperature over $20 \mathrm{~K}$.

One notable change regarding the cooling system design from the one explained in Ref. [4] is that we now separate the cooling paths for a test mass and radiation shields.
In the previous design, all of the four cryocoolers were connected to both the shields and the mirror. In the current design, the test mass suspension is connected to two cryocoolers and the radiation shields are connected to the other two. This way, the heat absorbed by the radiation shields coming from the large-angle scattering of the mirror surface does not affect the mirror temperature so much. This design allows us to use more laser power, which is different from the value used in Ref. [4].

\section{Mirror thermal noise}

The mirror thermal noise curve in Fig. 2 is the quadratic sum of substrate Brownian noise, coating Brownian noise, and substrate thermoelastic noise. Coating thermo-optic noise is supposed to be very low at $20 \mathrm{~K}$ and is ignored here. The formulas and the parameters used to calculate the thermal noises are given in Ref. [4].

\section{Suspension thermal noise}

Calculation of the suspension thermal noise is performed using a three-mass suspension system model consisting of a test mass, a penultimate mass, and a recoil mass suspended from the same penultimate mass [4]. The suspension materials for the penultimate mass and the recoil mass are tungsten and copper beryllium in this calculation.

The energy dissipation of a pendulum happens mainly at the top and bottom ends of the suspension fibers. We use the average temperature of the test and the penultimate masses as the effective temperature for the calculation of the thermal noise from the horizontal suspension modes. For the vertical modes, the effective temperature is not trivial. We use the average temperature along the suspension fiber for the calculation in this paper.

\section{OPTIMIZATION OF QUANTUM NOISE SHAPE}

\section{A. Quantum nondemolition techniques}

Quantum noises, i.e., shot noise and radiation-pressure noise, are mainly determined by input laser power, mirror reflectivities, and mirror masses. In addition to these 
parameters, we can modify the quantum noise shape and possibly beat the standard quantum limit (SQL) by using quantum nondemolition (QND) techniques. In KAGRA, we plan to use two QND techniques.

In order to extract the DARM signal, we use the DC readout scheme [6] to avoid the shot-noise penalty of the conventional RF readout scheme and for many other practical reasons [7]. In this scheme, a microscopic offset in DARM is introduced during the operation to leak a weak carrier field into the AS port. This carrier field serves as the local oscillator for the gravitational-wave sidebands (GWSBs) to generate power variation proportional to the gravitational-wave amplitude at the AS port. In reality, there is also some carrier light leaking to the AS port by the reflectivity difference of the two arm cavities. The relative phase of the local oscillator to the GWSB, called the homodyne angle $\zeta$, is determined by the amplitude ratio of these two carrier fields. Therefore, by adjusting the DARM offset, it is in principle possible to control the homodyne angle. When an appropriate value of $\zeta\left(\neq 90^{\circ}\right)$ is chosen, a cancellation of the shot noise and the radiationpressure noise happens, beating the SQL. This QND technique is called back-action evasion (BAE).

The second QND technique to be employed in KAGRA is an optical spring effect realized by detuning the SRC [8]. The detuning imposes a rotation of the GWSB phase at the reflection by the SRM. This induces differential radiation-pressure forces correlated to the GWSB on the test masses, amplifying the GW signal at certain frequencies. The parameter to characterize this QND scheme is the detuning angle $\phi$ of the SRC, which is defined by $\phi \equiv 2 \pi d / \lambda$, where $d$ is the deviation of the SRC length from the carrier resonance and $\lambda$ is the wavelength of the carrier light.

\section{B. Optimization of the mirror reflectivities}

The finesse $\mathcal{F}$ of the arm cavities and the reflectivity of the SRM $\left(R_{\mathrm{s}}\right)$ determine the quantum noise shape of an interferometer together with the homodyne angle $\zeta$ and the detuning angle $\phi$. These parameters are chosen by using the detection range of binary neutron star inspiral events (inspiral range $=I R$ ) as a figure of merit.

Figure 3 shows the inspiral range for $1.4_{\odot}-1.4_{\odot}$ neutron star binaries as functions of $\mathcal{F}$ with different values of $R_{\mathrm{s}}$. For each set of $\mathcal{F}$ and $R_{\mathrm{s}}, \zeta$ and $\phi$ are optimized to give the largest IR. The input power is adjusted to make the heat absorption of the test masses constant to keep the mirror temperature at $20 \mathrm{~K}$. We also plot the cases with $\phi=0$ to see the IRs for BRSE configurations. We assumed a round-trip loss of $100 \mathrm{ppm}$ for each arm cavity for this calculation.

As is evident from the plot, DRSE configurations give generally better inspiral ranges. However, a DRSE interferometer gives a narrower detection bandwidth than the BRSE configuration of the same mirror parameters. For

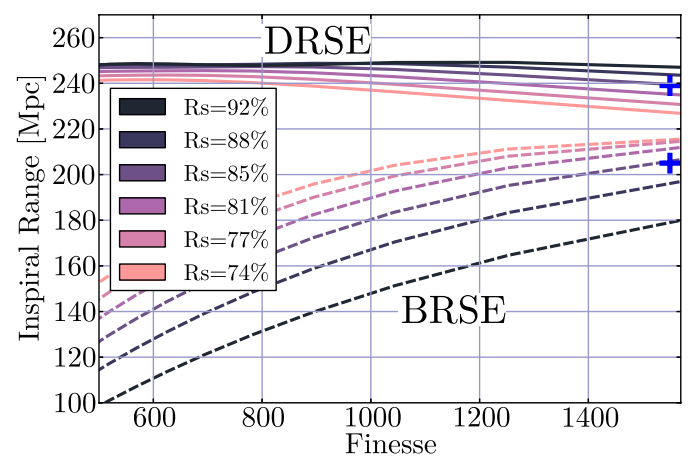

FIG. 3 (color online). Inspiral range with different finesse and signal-recycling mirror reflectivity.

example, in the case of Fig. 2, the sensitivity of BRSE is better than DRSE above $500 \mathrm{~Hz}$, where signals from the merger phase of a neutron star inspiral event are expected to appear $[9,10]$. This means, for the first detection, a DRSE interferometer gives us a better chance, while richer scientific information may be extracted from a BRSE detector. Moreover, the operation of a DRSE interferometer has some technical concerns, such as unwanted errorsignal offsets in the auxiliary d.o.f. by an imbalance of the RF sidebands used for signal extraction. Therefore, fixing the interferometer configuration to DRSE bears some risks. For these reasons, we decided to make our interferometer to be operated in both configurations (variable detuning). Variable detuning is realized by adding an offset into the error signal for the control of the SRC length. Therefore, the amount of possible detuning is limited to the linear range of the SRC error signal.

From Fig. 3, we select reflectivity parameters to have good IRs for both BRSE and DRSE. These are indicated by + marks in the plot. A higher finesse makes the interferometer susceptible to the losses of the mirrors. This trend can be seen in the DRSE curves. A smaller finesse decreases the BRSE sensitivity. Also, the optimal detuning angles of smaller-finesse cases are too large to be realized by the offset detuning method explained above. The value of $R_{\mathrm{S}}$ is selected to strike a balance between BRSE and DRSE. The PRM reflectivity is chosen to match the reflectivity of the arm cavities. Table II summarizes the selected reflectivities of the mirrors as well as the homodyne and the detuning angles of the KAGRA interferometer.

TABLE II. Parameters of the KAGRA interferometer related to the quantum noises.

\begin{tabular}{lccc}
\hline \hline Arm cavity finesse & 1530 & ITM reflectivity & $99.6 \%$ \\
ITM loss & $<50 \mathrm{ppm}$ & PRM reflectivity & $90 \%$ \\
ETM loss & $<50 \mathrm{ppm}$ & Homodyne angle & $132^{\circ}$ \\
SRM reflectivity & $85 \%$ & Input laser power & $78 \mathrm{~W}$ \\
Detuning angle & $3.5^{\circ}$ & DRSE IR & $237 \mathrm{Mpc}$ \\
BRSE IR & $217 \mathrm{Mpc}$ & & \\
\hline \hline
\end{tabular}




\section{LENGTH SENSING AND CONTROL}

In order to operate the interferometer with the quantumnoise-limited sensitivity discussed in the previous section, the interferometer mirrors have to be kept at certain operation states. For example, the arm-cavity lengths have to be kept at an integral multiple of the laser wavelength to resonate the light inside them. To achieve this, the positions and the orientations of the mirrors have to be monitored first. Then feedback control is used to keep them at the optimal operating points throughout the operation of the interferometer.

In this section, we discuss how to extract necessary information to control the mirrors. Although we have to control both the positions and the orientations of the mirrors, we only focus on the position (or length) control in this section. The alignment control is discussed in Sec. VII. The full account of the length signal extraction scheme is given in Ref. [11] and this section is a brief summary of that work.

\section{A. RF sideband resonant conditions}

We mainly use a variant of the RF readout scheme to extract the information of most of the d.o.f. that are to be controlled. Only the DARM signal is obtained using the DC readout scheme, as discussed in the Sec. IV.

Our sensing scheme makes use of RF sidebands, generated by phase modulations applied to the incident laser beam. Those RF sidebands generate beat signals against the carrier or other RF sidebands at the output ports of the interferometer. These beat signals contain information on the motions of the mirrors-usually mixtures of various d.o.f. In order to extract the information of the d.o.f. independently, the RF sidebands have to see different parts

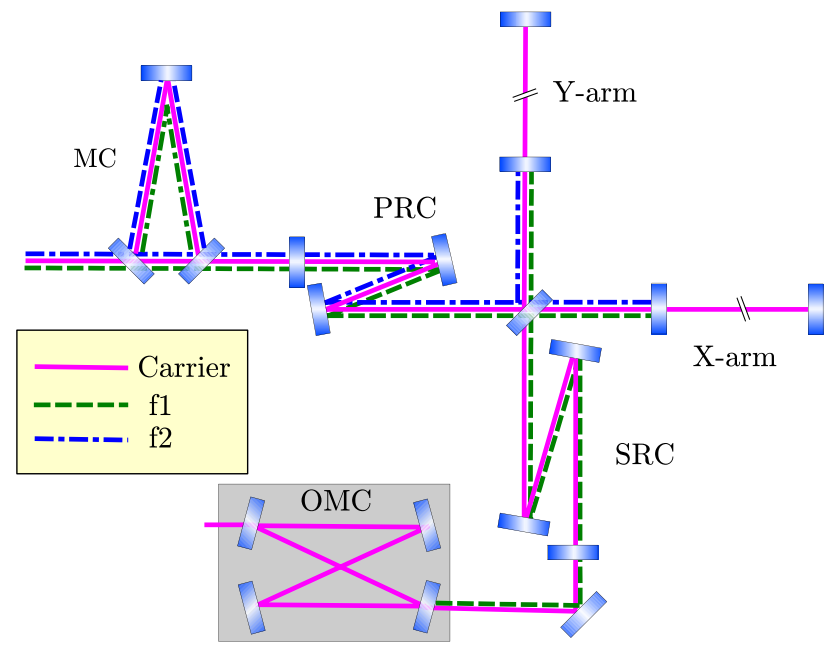

FIG. 4 (color online). Resonant conditions of the carrier and the RF sidebands. Each field is represented by lines of a distinct style. A field is resonant in the parts of the interferometer in which the corresponding lines are drawn. of the interferometer, i.e., they must resonate in different parts of the interferometer.

For the interferometer control of KAGRA, we use two sets of RF sidebands, called f1 and $\mathrm{f} 2$. The resonant conditions for those sidebands and the carrier inside the interferometer are depicted in Fig. 4. The carrier is resonant in the two arm cavities and the PRC. The AS side of the BS is kept at a dark fringe for the carrier. The $\mathrm{f} 1$ sidebands are resonant in the PRC and the SRC, but not in the arm cavities. The $\mathrm{f} 2$ sidebands resonate only in the PRC. In this way, we can expect these light fields to carry different information about the mirror motions. Since we apply the modulations before the MC, the two RF sidebands also have to resonate in the MC.

\section{B. Modulation frequencies and macroscopic lengths}

The resonant conditions of the RF sidebands are determined by the macroscopic lengths of the PRCs and the SRCs and the macroscopic asymmetry of the $\mathrm{MICH}$, as well as the RF modulation frequencies. There are many combinations of these parameters which can realize the resonant conditions explained above. However, we have to satisfy several practical constraints when choosing them.

First of all, it is desirable to have short PRC and SRC from the viewpoint of construction cost, especially in the underground site of KAGRA. However, the PRC and SRC lengths have to be long enough to house the Z-shaped folding part without causing too much astigmatism on the laser beams. In addition, we have to include $20 \mathrm{~m}$-long cold sections in the vacuum pipes between the ITMs and the BS to prevent the room-temperature thermal radiation from bombarding the cold test masses. The RF modulation frequencies are constrained to below $50 \mathrm{MHz}$ from the available response speed of photodetectors with large apertures. It is also desirable to be above $10 \mathrm{MHz}$ to avoid large low-frequency laser noises.

We tested a large number of combinations of the length and the frequency parameters to find ones which satisfy the resonant conditions and the practical constraints at the same time. Out of several surviving candidates, we chose the parameters shown in Table III. We used the loop noise coupling estimates, explained in the next section, to decide the best parameter sets [11].

\section{Fine-tuning of the RF sideband frequencies}

The RF sidebands $\mathrm{f} 1$ and $\mathrm{f} 2$ are almost antiresonant to the arm cavities, but not perfectly so. A consequence of this

TABLE III. Length and frequency parameters. The values shown here are after the adjustment of Sec. VC.

\begin{tabular}{lccc}
\hline \hline Arm cavity length & $3000 \mathrm{~m}$ & f1 frequency & $16.881 \mathrm{MHz}$ \\
PRC length & $66.591 \mathrm{~m}$ & f2 frequency & $45.016 \mathrm{MHz}$ \\
SRC length & $66.591 \mathrm{~m}$ & MC length & $26.639 \mathrm{~m}$ \\
Michelson asymmetry & $3.30 \mathrm{~m}$ & & \\
\hline \hline
\end{tabular}


is that they get small but finite phase shifts when reflected by the arm cavities. These two sidebands have to resonate in the PRC at the same time. However, if the phase shifts they get from the arm cavities are arbitrary, the resonant conditions for them are different, and thus we cannot resonate both of them at the same time. For this reason, a fine-tuning of the RF sideband frequencies is necessary.

An effective cavity-length change caused by a phase shift $\phi$ for a modulation sideband with a modulation frequency $\omega_{\mathrm{m}}$ is $\Delta L=\phi c / \omega_{\mathrm{m}}$. Therefore, if the phase shifts for the $\mathrm{f} 1$ and $\mathrm{f} 2$ sidebands are proportional to their frequencies, the effective length change is the same for the two SBs. Then we can just pre-shorten the PRC length by this amount to fulfill the resonant conditions for both of the sidebands at the same time.

In order to adjust the reflection phases for $\mathrm{f} 1$ and $\mathrm{f} 2$, we need to change their frequencies relative to the carrier resonance. However, we have to keep the ratio of $\mathrm{f} 1$ and f2 frequencies to be 3:8 to fulfill the resonant conditions of Fig. 4 with the macroscopic length parameters of Table III. This is automatically satisfied by requiring the two sidebands to transmit the $\mathrm{MC}$, i.e., the $\mathrm{f} 1$ frequency is 3 times the free spectral range (FSR) of the $\mathrm{MC}$ and $\mathrm{f} 2$ is 8 times the MC FSR. Therefore, we will slightly change the MC length from its nominal value to find the optimal RF sideband frequencies which give the desired arm-cavity reflection phases. The precise amount of the phase shifts induced on nearly antiresonant fields by a cavity depends on its finesse. Therefore, the RF sideband frequencies must be adjusted according to the measured value of the real arm-cavity finesse. In this paper, we assume $100 \mathrm{ppm}$ of loss in the arm, resulting in a finesse of 1530 . Figure 5 shows the ratio of the reflection phases $\left(\phi_{2} / \phi_{1}\right)$ as a function of the MC length. The desired value of $8 / 3$ is indicated by a green horizontal line. By finding an intersection of the blue curve with the green line, tentative numbers for the RFSB frequencies are determined to be $\mathrm{f} 1=16.881 \mathrm{MHz}$ and $\mathrm{f} 2=45.016 \mathrm{MHz}$. Corresponding changes of the PRC and the SRC lengths are $5.7 \mathrm{~mm}$ and $11.4 \mathrm{~mm}$, respectively.

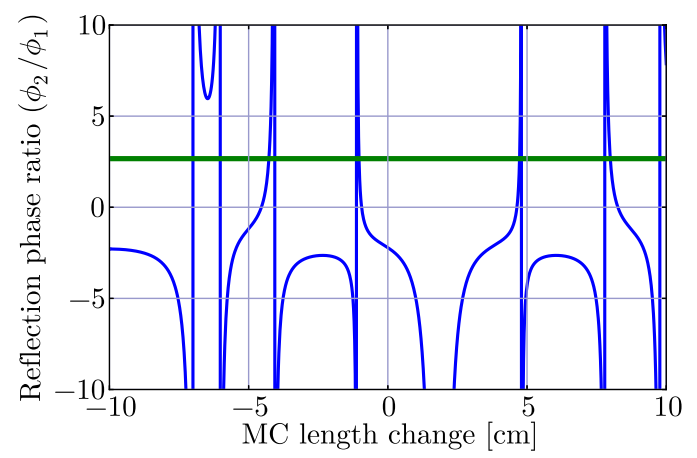

FIG. 5 (color online). Ratio of the RF sideband reflection phases by the arm cavities. We want to set it to $8 / 3$, which is indicated by the green line.

\section{Sensing matrix}

Once a parameter set is chosen for the macroscopic length and the modulation frequencies, we can calculate the response of the interferometer, that is, how much beat signals at each detection port are changed in response to the motion of the mirrors. We use an interferometer simulation tool, called OPTICKLE [12], for this calculation. By solving self-consistent equations of optical fields inside the interferometer, OPTICKLE computes the strength of the beat signals at each detection port. OPTICKLE also takes into account the radiation-pressure effect to correctly compute the response of a high-power interferometer.

There are three detection ports and two primary beat frequencies ( $\mathrm{f} 1$ and $\mathrm{f} 2$ ). For each beat frequency, there is a choice of two orthogonal demodulation phases. Therefore, there are $3 \times 2 \times 2=12$ candidate signals to be used as error signals. Out of these signals, we chose ones with good signal strength and separation between d.o.f. The signal-sensing matrices of the KAGRA interferometer with the selected parameters and signal ports are shown in Tables IV and V. These matrices are frequency dependent. The values shown in the tables are calculated at $100 \mathrm{~Hz}$.

\section{E. Loop noise coupling}

The sensing matrices shown in Tables IV and V are clearly not diagonal. Therefore, feedback control using these signals causes some cross-couplings between the d.o.f. Since each error signal has its own noises, the cross-couplings could inject excess noises from the auxiliary d.o.f. into the DARM signal. This mechanism is called loop noise coupling [13]. Along with the interferometer response to the mirror motions, OPTICKLE can also compute the quantum noise at each signal port. Using this information, we can compute the amount of loop noise couplings.

For the calculation of the loop noise, we have to assume the shape of open-loop transfer functions of the servo loops. We assume a simple $1 / f^{2}$-shaped transfer function with $1 / f$ response around the unity gain frequency (UGF) for stability. The UGFs are set to $200 \mathrm{~Hz}$ for DARM, $10 \mathrm{kHz}$ for CARM, and $50 \mathrm{~Hz}$ for all the other d.o.f.

The calculated loop noise couplings to the DARM signal are shown in Fig. 6. Obviously, the injected noises from the auxiliary d.o.f. are too high and compromise the target sensitivity. However, we can mitigate this problem by using a technique called feed-forward [11], which was widely used in the first-generation interferometric detectors. A loop noise is first injected into an auxiliary mirror, such as the PRM, by a feedback force. Then the noiseinduced motion of this mirror is coupled to the DARM signal by the off-diagonal elements of the sensing matrix. This means that by knowing how much force is applied to the auxiliary mirrors, and by experimentally measuring the off-diagonal elements of the sensing matrix, we can precisely estimate the noise signal injected into DARM. Then, we can subtract this noise either by signal processing or by 
TABLE IV. Length-sensing matrix for BRSE. AS_DC: DC readout signal at the AS port. REFL_f1I: REFL signal demodulated at the f1 frequency in in-phase. REFL_f1Q: REFL signal demodulated at the f1 frequency in quadrature-phase. POP_f1I and POP_f2I: POP signal demodulated in in-phase at the $\mathrm{f} 1$ and the $\mathrm{f} 2$ frequencies, respectively. The values are the transfer coefficients at $100 \mathrm{~Hz}$ from the motion of the mirrors to the signal ports, with each row normalized by the diagonal element.

\begin{tabular}{lccccc}
\hline \hline & DARM & CARM & MICH & PRCL & SRCL \\
\hline AS_DC & 1 & $4.2 \times 10^{-5}$ & $1.0 \times 10^{-3}$ & $4.8 \times 10^{-6}$ & $4.7 \times 10^{-6}$ \\
REFL_f1I & $5.4 \times 10^{-3}$ & 1 & $4.3 \times 10^{-5}$ & $6.5 \times 10^{-3}$ & $4.3 \times 10^{-3}$ \\
REFL_f1Q & $5.0 \times 10^{-3}$ & $1.3 \times 10^{-2}$ & 1 & 1.02 & 0.67 \\
POP_f2I & $2.3 \times 10^{-2}$ & 4.3 & $1.0 \times 10^{-2}$ & 1 & $2.5 \times 10^{-4}$ \\
POP_f1I & $8.7 \times 10^{-2}$ & 16.2 & $3.1 \times 10^{-2}$ & 2.1 & 1 \\
\hline \hline
\end{tabular}

TABLE V. Length-sensing matrix for DRSE. REFL_f2I: REFL signal demodulated at the f2 frequency in in-phase.

\begin{tabular}{lccccc}
\hline \hline & DARM & CARM & MICH & PRCL & SRCL \\
\hline AS_DC & 1 & $4.1 \times 10^{-5}$ & $1.0 \times 10^{-3}$ & $4.5 \times 10^{-6}$ & $7.6 \times 10^{-6}$ \\
REFL_f2I & $1.2 \times 10^{-2}$ & 1 & $1.3 \times 10^{-4}$ & $1.2 \times 10^{-2}$ & $1.4 \times 10^{-3}$ \\
REFL_f1Q & $2.8 \times 10^{-2}$ & $9.9 \times 10^{-3}$ & 1 & 0.39 & 0.18 \\
POP_f2I & $2.7 \times 10^{-2}$ & 4.3 & $1.0 \times 10^{-2}$ & 1 & $8.5 \times 10^{-5}$ \\
POP_f1I & $1.7 \times 10^{-1}$ & 35 & $3.1 \times 10^{-2}$ & 2.0 & 1 \\
\hline \hline
\end{tabular}

feed-forwarding the estimated noise to the DARM actuators with opposite sign.

Figure 7 shows loop noise couplings after the feedforward scheme is applied. We assume that the noise cancellation is performed with $1 \%$ accuracy. This result assures that the signal-sensing scheme and the parameters we selected yield sufficiently low-noise signals for the length control of the interferometer. In the design process, we repeatedly computed the loop-noise plots (like Fig. 7) with various interferometer parameters to choose the best set of the parameters.

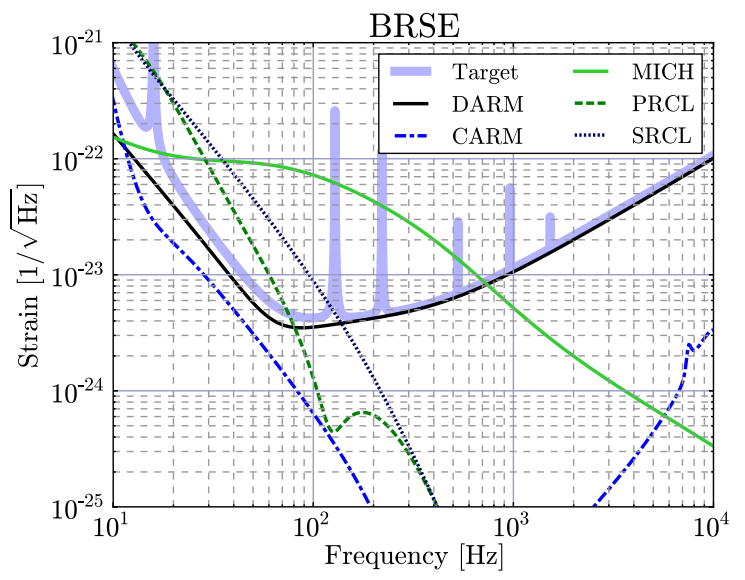

FIG. 6 (color online). Loop noise couplings.

\section{SPATIAL OPTICAL MODES}

Up to this point, we have analyzed the interferometer with the scalar field approximation, disregarding the spatial-mode shape of the laser beams. However, in a real interferometer, we have to mode-match the various parts of the interferometer to resonate only the necessary optical mode, i.e., the TEM00 mode. In this section, we consider the spatial-mode design of the interferometer with the goal of determining the radii of curvature of the interferometer mirrors.

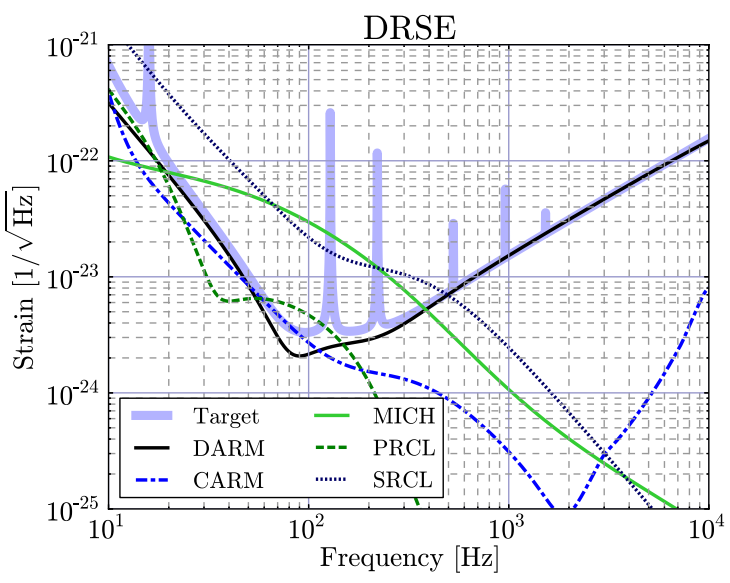



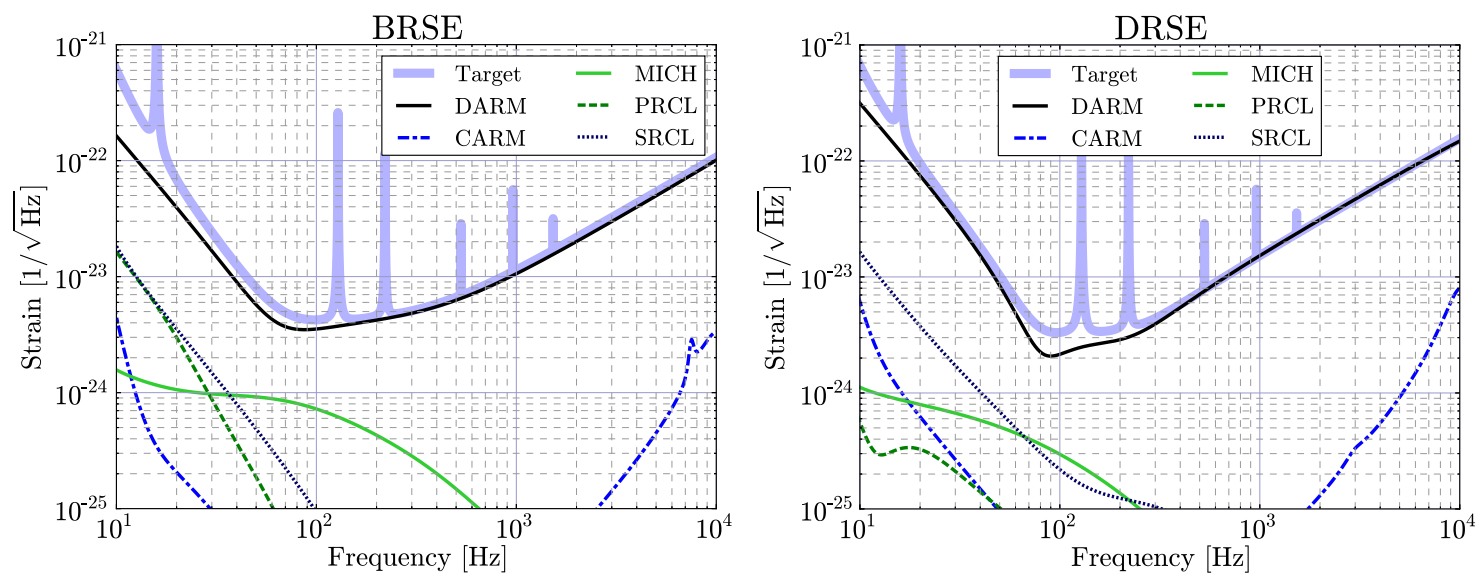

FIG. 7 (color online). Loop noise couplings with feed-forward.

\section{A. Arm cavities}

\section{1. $g$-factor}

The spatial-mode characteristics of a Fabry-Perot cavity are determined by the g-factors, which are defined as follows:

$$
g=g_{1} \cdot g_{2}, \quad g_{1} \equiv 1-\frac{L}{R_{1}}, \quad g_{2} \equiv 1-\frac{L}{R_{2}},
$$

where $L$ is the length of the cavity and $R_{1}$ and $R_{2}$ are the ROCs of the ITM and the ETM. The g-factors determine the beam-spot sizes on the mirrors and the degree of degeneracy of the higher-order spatial modes in the cavity.

First, we consider the beam-spot sizes on the mirrors, because they directly affect the noise of the interferometer through the thermal noise coupling. We want to make the beam sizes as large as possible to reduce mirror thermal noises. If we assume $R_{1}=R_{2}$, the beam-spot size $w$ is the same on both the mirrors, and it can be written as a function of the common g-factor $g_{0} \equiv g_{1}=g_{2}$,

$$
w=\sqrt{\frac{\lambda L}{\pi} \sqrt{\frac{1}{\left(1+g_{0}\right)\left(1-g_{0}\right)}}} .
$$

Since this is an even function of $g_{0}$, there are two possible values of $g_{0}$ giving the same spot size.

The high optical power circulating inside KAGRA's arm cavities generates strong angular optical spring effects [14]. There are always two angular spring modes: one is a positive-spring and the other is a negative-spring mode. The negative-spring mode causes angular instability of the mirrors if it is stronger than the mechanical restoring force of the mirror suspension. It is known that for the same beam size, the negative-spring constant is made smaller by choosing a negative g-factor $\left(g_{0}\right)$. Therefore, we prefer a negative value of $g_{0}$.

For our $22 \mathrm{~cm}$-diameter mirrors, the maximum possible beam size is $4.0 \mathrm{~cm}$, requiring the diffraction loss per reflection to be less than $1 \mathrm{ppm}$. The negative $g_{0}$ to realize this spot size is -0.772 , corresponding to a mirror ROC of $1692 \mathrm{~m}$. However, because of the time and cost constraints, we have to choose an ROC which can be polished using one of the stock reference spheres of the polishing company. For this reason, we have to change the ROC to $1900 \mathrm{~m}$. This reduces the beam-spot size to $3.5 \mathrm{~cm}$. The thermal noise increase due to this change degrades the IR from $221 \mathrm{Mpc}$ to $217 \mathrm{Mpc}$ for BRSE and from $243 \mathrm{Mpc}$ to $237 \mathrm{Mpc}$ for DRSE.

\section{Carrier higher-order mode resonances}

Ideally, the arm cavities should resonate only the TEM00 mode during the operation. However, optical higher-order modes (HOMs) are not completely antiresonant to the arm cavity in general. Therefore, if there is misalignment or mode mismatching, HOMs could resonate in the arm cavities, potentially increasing the shot noise. If the selected arm g-factor is a particularly bad one, this HOM coupling could be large. In this section, we confirm that our g-factor does not allow excessively large resonances of HOMs.

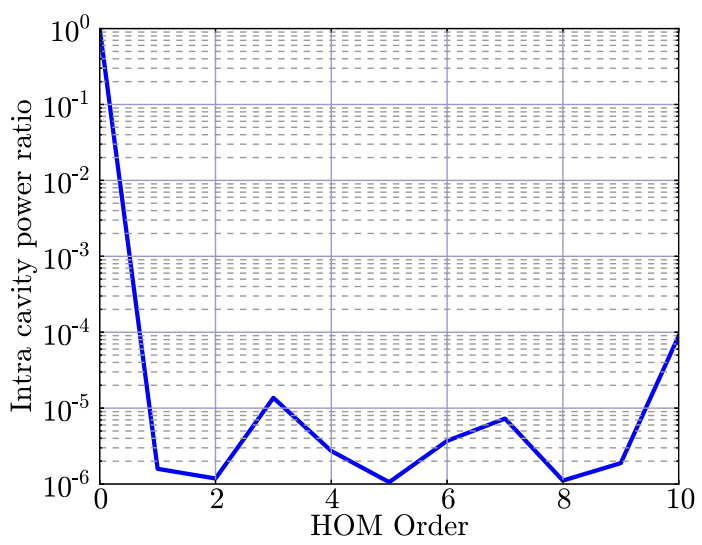

FIG. 8 (color online). HOM power in the arm cavity relative to the TEM00 power. The mode number is $n+m$ for TEMnm modes. 
INTERFEROMETER DESIGN OF THE KAGRA ...

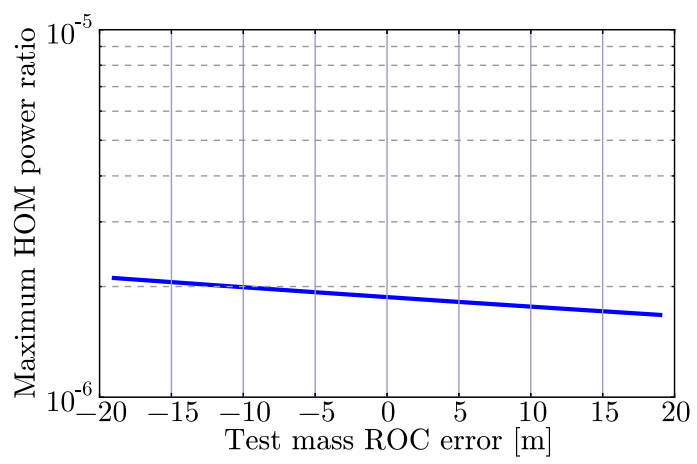

FIG. 9 (color online). The maximum HOM power ratio in the arm cavity as a function of the test mass ROC error. The ROC is swept by $\pm 1 \%$ around the nominal value.

Figure 8 shows the HOM power ratio to the TEM00 power in an arm cavity. This is the ratio of the intra-cavity optical power, if TEM00 and HOM modes are injected to the arm cavity with the same power. When calculating an HOM power, we take into account the fact that for HOMs the diffraction loss is higher than for TEM00. This is because HOMs are spatially spread more widely. The diffraction losses were calculated with the FFT optical simulation tool SIS [15].

Figure 8 assumes that the g-factor of the cavity is exactly as designed. In reality, there is always some error in the ROCs of real mirrors. We set the error tolerance to be $\pm 0.5 \%$ mainly from the technical feasibility of mirror polishing. Figure 9 shows the maximum HOM power ratio (the value of the highest peak in Fig. 8 except for the mode number $=0$ ) as a function of ROC error. There is no significant change in the HOM power ratio throughout the error range. This means that our g-factor is robust against mirror-fabrication errors.

\section{3. $R F$ sideband higher-order resonances}

Although the RF sideband frequencies are chosen to be nonresonant to the arm cavities for the TEM00 mode, their HOMs may accidentally hit a resonance of the arm cavities. This can cause an unwanted coupling of armcavity alignment fluctuations to the error signals of the auxiliary d.o.f.

Figure 10 shows the positions of the RF sidebands and their HOMs in the FSR of the arm cavity. In the figure, both the HOM resonant curves (Lorentzian-shaped curves with mode numbers) and the frequencies of the RF sidebands (vertical lines) are shown. We can see that there is no significant overlap between the RF sidebands and the HOM resonances.

In reality, the exact frequencies of the RF sidebands change according to the fine-tuning, as explained in Sec. VC. Therefore, accidental coincidences of an RF sideband and an HOM resonance could still happen. In this case, we can try to use a different crossing point from Fig. 5 to move the RF
PHYSICAL REVIEW D 88, 043007 (2013)

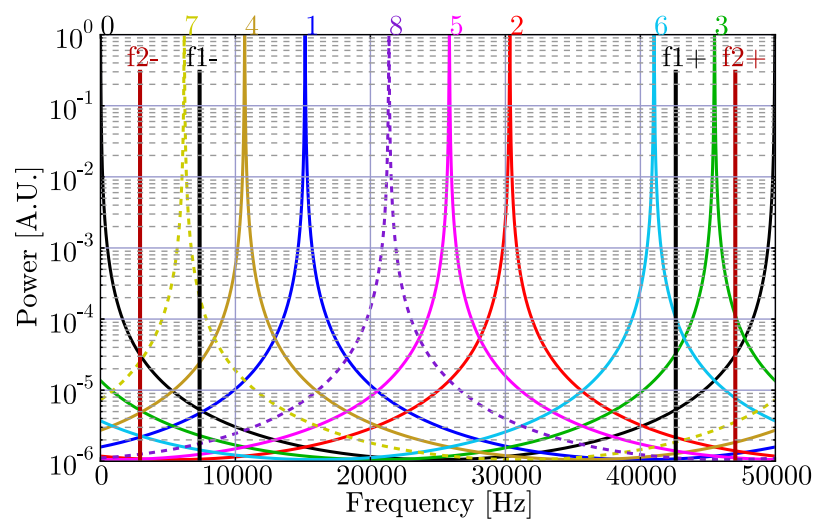

FIG. 10 (color online). Positions of the RF SBs and the HOMs in an FSR of the arm cavities. The colorful sharp peaks represent the resonant curves of the HOMs. The mode numbers are printed at the top of each resonance. The vertical lines are the positions of the RF SBs. The "+" and "-" signs indicate upper and lower sidebands, respectively.

sideband frequencies away from problematic HOM resonances and avoid unfortunate overlaps.

\section{B. Recycling cavities \\ 1. Gouy-phase shifts}

Now we turn our attention to the recycling cavities. The spatial-mode characteristic of the recycling cavities can be determined by the Gouy-phase changes of the light along the optical paths of the cavities. Because the arm cavities are very long, if we use a straight recycling cavity and inherit the spatial mode of the arm cavities without modification, the one-way Gouy-phase change inside the recycling cavity is very small (less than $1^{\circ}$ ). This makes the cavity highly degenerated for HOMs. Therefore, small alignment fluctuations or thermal lensing can cause the excitation of HOMs inside the recycling cavities. This is especially a problem for the RF sidebands, which only resonate in the recycling cavities and do not receive a mode-healing effect from the stable arm cavities. The consequence is poor spatial-mode overlap between the RF sidebands and the carrier, resulting in increased shot noise for the error signals of the auxiliary d.o.f. This was one of the most serious problems that the first-generation interferometers struggled against.

In order to avoid the degenerated recycling-cavity problem, we want to increase the Gouy-phase shifts in the recycling cavities. For this purpose, we fold the cavities with two additional mirrors, as shown in Fig. 1. Before going into the details of the folding scheme, we first discuss the desired values of one-way Gouy-phase changes in the PRC $\left(\eta_{\mathrm{p}}\right)$ and the $\operatorname{SRC}\left(\eta_{\mathrm{s}}\right)$.

\section{Higher-order mode power in the PRC}

Figure 11 shows a two-dimensional map of the HOM degeneracy in the PRC. Each point in the map represents a 


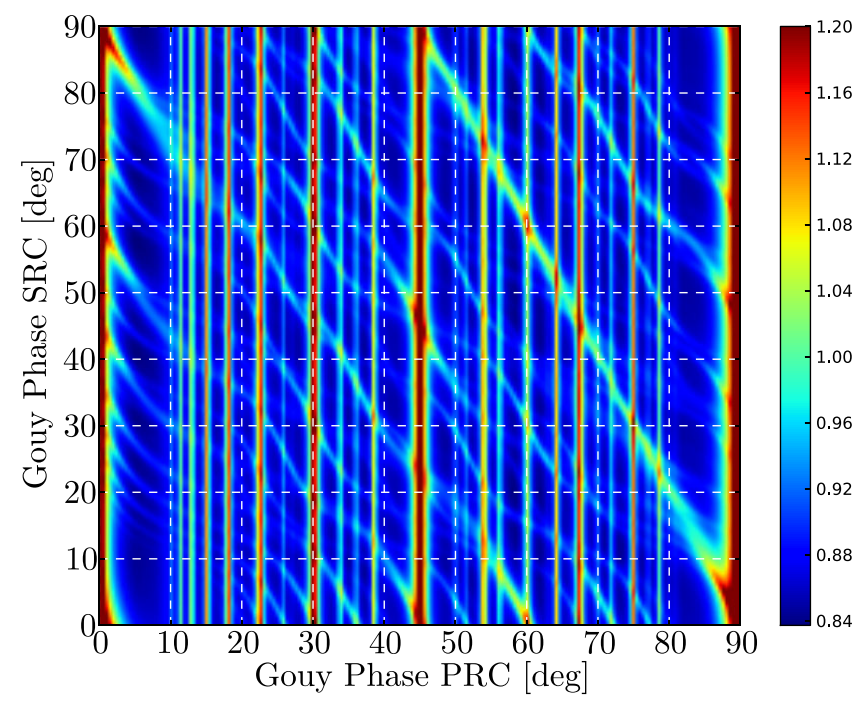

FIG. 11 (color online). Higher-order mode resonance map of the PRC.

combination of $\left(\eta_{\mathrm{p}}, \eta_{\mathrm{s}}\right)$. The color-coded value at each point represents the severity of the HOM degeneracy and it is computed as follows. Assuming an input laser power of $1 \mathrm{~W}$ at the carrier frequency injected from the back of the PRM, we compute the light power circulating in the PRC by solving the static field equations of the interferometer. We repeat this calculation by changing the mode of the input beam from the TEM00 mode to HOMs of up to the 15 th order. We then take the sum of the computed intraPRC power of the HOMs and normalize it with the power of the TEM00 mode. If some of the HOMs are close to the resonance in the PRC, this value (called $\zeta_{\mathrm{c}}$ ) becomes large. This process is also repeated for the $\mathrm{f} 1$ and the $\mathrm{f} 2$ sidebands, yielding the ratios $\zeta_{\mathrm{f} 1}$ and $\zeta_{\mathrm{f} 2}$. The color-coded value in the map of Fig. 11 is the sum of $\zeta_{\mathrm{c}}, \zeta_{\mathrm{f} 1}$, and $\zeta_{\mathrm{f} 2}$.

There are several dark areas in the map of Fig. 11. We want to select $\eta_{\mathrm{p}}$ and $\eta_{\mathrm{s}}$ centered in one of these large dark areas. We do not take the lower left area centered around $\left(\eta_{\mathrm{p}}, \eta_{\mathrm{s}}\right)=\left(5.5^{\circ}, 8^{\circ}\right)$, because this region gives too much degeneracy in the SRC, as explained in the next section. In order to select a preferred parameter set from the other candidate areas, we computed the wave-front sensing signals (Sec. VII) and their diagonalized shot noises [Eq. (5)] repeatedly with all the candidate parameters. As a consequence, we arrive at $\eta_{\mathrm{p}}=16.5^{\circ}$ and $\eta_{\mathrm{s}}=17.5^{\circ}$ as the parameter set to give the lowest shot noises. The dark region around this parameter set is not so large compared with other dark areas. However, the area is sufficiently large considering the error tolerance of the ROCs of the folding mirrors, as discussed in Sec. VIB 5.

\section{Degeneracy of the SRC}

In the previous section, we basically computed the HOM resonances in the coupled PRC-SRC for the fields injected from the PRM. However, there is one important process which requires a separate treatment, involving the degeneracy of the SRC. When there is a gravitational wave passing through the detector, GWSBs are excited in the arm cavities with opposite phases. These GWSBs come out of the antisymmetric side of the BS and are reflected by the SRM. If there is some figure error or defects on the surface of the SRC mirrors, the GWSBs, which are in the TEM00 mode defined by the arm cavities, can be scattered into HOMs inside the SRC. Although the amount of the scattering by high-quality mirrors is expected to be very small, if one of the HOMs is resonant in the SRC, the scattering loss is significantly enhanced [16], causing the reduction of the net GW signal. This process can be investigated by injecting a laser beam from the back of the SRM and checking the HOM resonances.

We use the same field equations used in the previous section to compute the SRC degeneracy, but inject the input beam from the back of the SRM this time. The SRC length is controlled to be resonant to the carrier by itself. However, when the arm cavities are locked, the carrier gets an extra sign flip at the back of the ITMs. Therefore, they are not resonant in the SRC when the field is injected from the SRM side. On the other hand, the HOMs of the carrier are not resonant in the arm cavities, receiving no sign flip from them. Therefore, a degenerate SRC can resonate HOMs. Because of this resonant conditions, it does not make sense to normalize the HOM powers with the power of the TEM00 mode for this study. Instead, we first compute the power of the $n$th HOM, $P_{\mathrm{d}}(n)$, in the SRC when it is completely degenerated, i.e., $\eta_{\mathrm{s}}=0$. Then we calculate the same HOM power values, $P_{\mathrm{s}}(n)$, with a finite value of $\eta_{\mathrm{s}}$. For each HOM, we take the ratio of the intra-SRC power $\xi_{n}=$ $P_{\mathrm{s}}(n) / P_{\mathrm{d}}(n)$. This ratio represents how much the resonantly enhanced scattering problem is relieved by adding a finite Gouy-phase shift to the SRC.

Figure 12 shows the computed $\xi_{n}$ as a function of the HOM order $n$ for $\left(\eta_{\mathrm{p}}, \eta_{\mathrm{s}}\right)=\left(16.5^{\circ}, 17.5^{\circ}\right)$. For all the HOMs computed, $\xi_{n}$ is smaller than 1 , meaning the HOM resonance is reduced from the completely

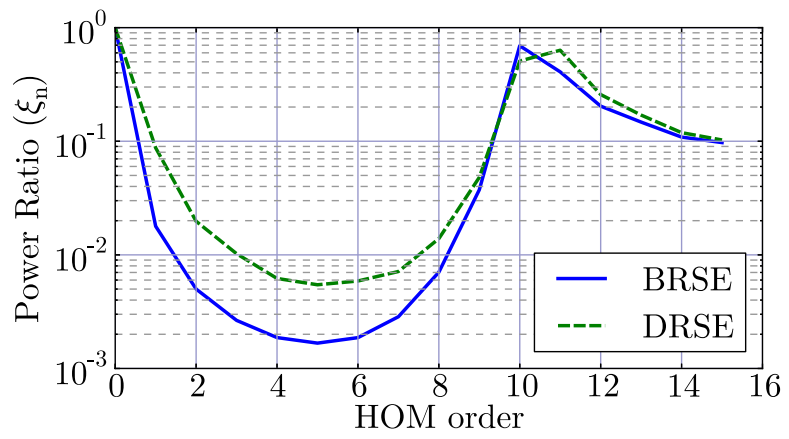

FIG. 12 (color online). Higher-order mode resonance scan of the SRC for $\left(\eta_{\mathrm{p}}, \eta_{\mathrm{s}}\right)=\left(16.5^{\circ}, 17.5^{\circ}\right)$. 


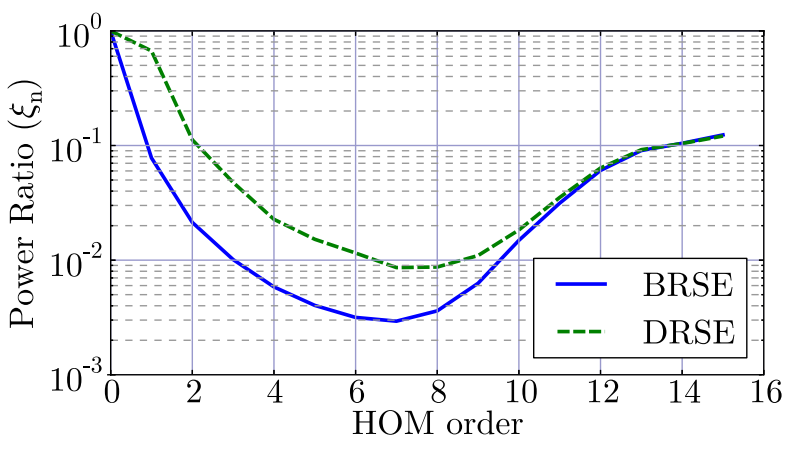

FIG. 13 (color online). Higher-order mode resonance scan of the SRC for $\left(\eta_{\mathrm{p}}, \eta_{\mathrm{s}}\right)=\left(5.5^{\circ}, 8^{\circ}\right)$.

degenerated case. At orders 10 and 11, the reduction is not so large. However, we should note that because of the diffraction loss, the finesse of the SRC is reduced by a factor of 3 for these HOMs.

Figure 13 shows the same HOM scan for $\left(\eta_{\mathrm{p}}, \eta_{\mathrm{s}}\right)=$ $\left(5.5^{\circ}, 8^{\circ}\right)$ with DRSE. The first HOM is not well suppressed, especially for DRSE. This is a problem because the first-order modes are strongly coupled with mirror alignment fluctuations and are easily excited. For this reason, we did not employ the small Gouy-phase regions of Fig. 11.

\section{Gouy-phase telescopes in the recycling cavities}

In order to realize the desired Gouy-phase shifts in the recycling cavities, we have to focus the beams inside the cavities. We achieve this by folding the cavities with two additional mirrors (folding mirrors) to form a telescope. The schematic of the folding part of the PRC is shown in Fig. 14. Although we mainly use the PRC for the explanation in this section, the design for the SRC is almost identical to the PRC.

The total length of the cavity has to be equal to the one determined in Sec. V B. In order to reduce the astigmatism, we want to separate the PR2 and the PR3 as much as possible. With other practical constraints (mainly the size of the vacuum chambers), we set the lengths of the folding part as shown in Table VI.

After selecting the separations between the folding mirrors, we optimize the ROCs of the folding mirrors. There

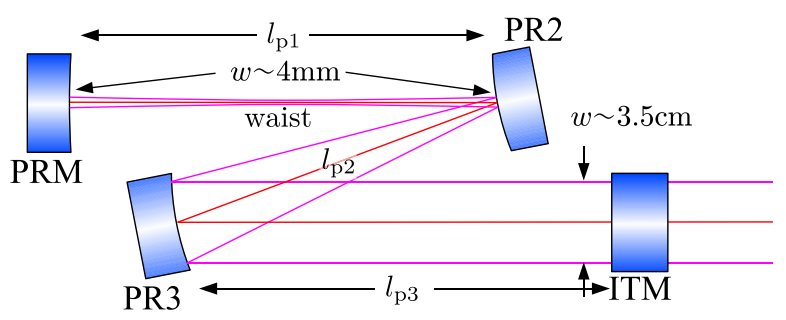

FIG. 14 (color online). Schematic view of the folded powerrecycling cavity. The BS is omitted in this figure and the ITMs are combined into a single effective ITM.
TABLE VI. Parameters of the folding cavities. $l_{\mathrm{p} 1}, l_{\mathrm{p} 2}$, and $l_{\mathrm{p} 3}$ are the lengths of the three segments of the folded PRC as shown in Fig. 14. $l_{\mathrm{s} 1}, l_{\mathrm{s} 2}$, and $l_{\mathrm{s} 3}$ are the corresponding lengths of the SRC.

\begin{tabular}{lllc}
\hline \hline$l_{\mathrm{p} 1}$ & $14.762 \mathrm{~m}$ & PRM ROC & $458.129 \mathrm{~m}$ \\
$l_{\mathrm{p} 2}$ & $11.066 \mathrm{~m}$ & PR2 ROC & $-3.076 \mathrm{~m}$ \\
$l_{\mathrm{p} 3}$ & $15.764 \mathrm{~m}$ & PR3 ROC & $24.917 \mathrm{~m}$ \\
$l_{\mathrm{s} 1}$ & $14.741 \mathrm{~m}$ & SRM ROC & $458.129 \mathrm{~m}$ \\
$l_{\mathrm{s} 2}$ & $11.112 \mathrm{~m}$ & SR2 ROC & $-2.987 \mathrm{~m}$ \\
$l_{\mathrm{s} 3}$ & $15.739 \mathrm{~m}$ & SR3 ROC & $24.917 \mathrm{~m}$ \\
\hline \hline
\end{tabular}

are many combinations of ROCs to realize a given Gouyphase shift in the PRC. The selected values of the ROCs are shown in Table VI. Figure 14 shows the beam profile of the PRC with the selected ROCs. The beam from the ITMs is focused by the PR3 and hits the PR2 with a smaller beam size. The PR2 is a convex mirror used to collimate the beam. There is a beam waist at the middle of the PR2 and the PRM. Therefore, this design gives the same beam-spot size on the PRM and the PR2. Other combinations of the ROCs giving the same Gouy-phase change in the PRC make the beam-spot sizes larger on one mirror and smaller on the other compared with our design. A smaller spot size increases the concern for thermal lensing. A larger beam makes it harder to handle the beams at the REFL and the POP ports. The $4 \mathrm{~mm}$ beam, which is also well collimated, can be easily steered with standard 2 inch optics. The small beam size also makes it easy to separate secondary reflection beams from, for example, the antireflection surfaces of the ITMs. These stray beams have to be properly damped to avoid scattered light noises. The large beams coming back from the arm cavities ( $3.5 \mathrm{~cm}$ radius) are not easy to separate. We utilize the beam-reducing functionality of the Gouy-phase telescope as an extra benefit to cleanly separate the stray beams between the PR2 and the PRM after the beam sizes are reduced.

Although the desired values of the Gouy-phase shifts in the PRC and the SRC are slightly different, we decided to use almost the same ROCs for the folding mirrors of the two cavities, so that the same reference spheres can be used for polishing. We slightly changed the length parameters of the SRC from the PRC to realize a different Gouy-phase shift with only a minimal change of the ROC of the SR2. The total length of the SRC is not changed with this adjustment.

Table VII shows expected thermal lens effects on the PRC mirrors. The following formula is used to asses the effective change of the ROC by thermal lensing [17]:

$$
d R=\frac{\alpha R^{2} P_{\mathrm{a}}}{2 \pi \kappa w^{2}}
$$

where $R$ is the ROC of the mirror, $\alpha$ is the thermal expansion coefficient of the substrate, $\kappa$ is the thermal conductivity, $w$ is the beam-spot radius on the mirror, 
TABLE VII. Thermal lens effect on the PRC mirrors.

\begin{tabular}{lccc}
\hline \hline Mirror & Beam radius & $d R$ & Tolerance \\
\hline PRM & $4 \mathrm{~mm}$ & $5.5 \mathrm{~m}$ & $\pm 20 \mathrm{~m}$ \\
PR2 & $4 \mathrm{~mm}$ & $0.24 \mathrm{~mm}$ & $\pm 10 \mathrm{~mm}$ \\
PR3 & $35 \mathrm{~mm}$ & $0.21 \mathrm{~mm}$ & $\pm 10 \mathrm{~mm}$ \\
\hline \hline
\end{tabular}

and $P_{\mathrm{a}}$ is the absorbed light power at the surface of the mirror. We assume an intra-cavity power of $800 \mathrm{~W}$ and $10 \mathrm{ppm}$ absorption loss at the reflection of each mirror. Although the $10 \mathrm{ppm}$ absorption is rather large, we take it as a safety margin. The amount of the ROC change from the thermal lensing is less than the figure error tolerance discussed in the next section.

\section{ROC error}

The mode profile of the Gouy-phase telescope is highly sensitive to the errors in the ROCs of the mirrors, especially of the PR2 and the PR3. If the mode of the PRC is not matched with the arm-cavity modes, the recycling gain is reduced. In the case of mode mismatch between the SRC and the arm cavities, the gravitational-wave sidebands coming out to the AS port are reduced. In addition, a mode profile change is usually associated with a deviation of the Gouy-phase shift from the desired value.

Figure 15 shows the mode-mismatching value and the deviation of the one-way Gouy-phase shift from the desired value, plotted against the error in the ROCs of PR2 and PR3. The mode-mismatching value is defined by the following formula:

$$
1-\left|\int_{-\infty}^{\infty} \int_{-\infty}^{\infty} \psi_{\mathrm{PRC}}^{*}(x, y) \psi_{\mathrm{ARM}}(x, y) d x d y\right|^{2},
$$
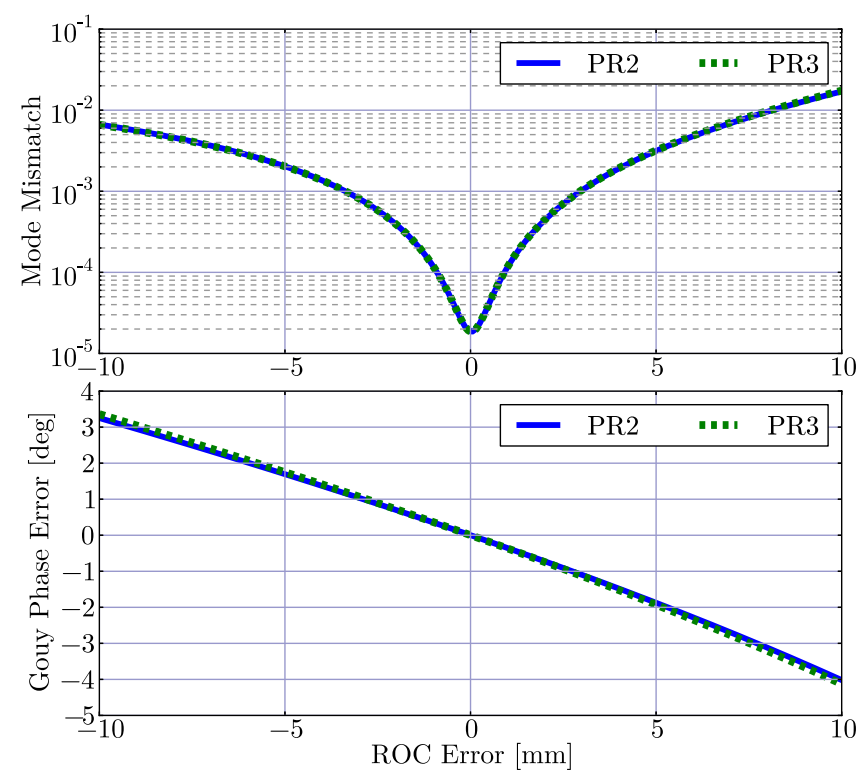

FIG. 15 (color online). The mode mismatch and the Gouyphase error as functions of the ROC errors of the PR2 and the PR3. where $\psi_{\text {PRC }}(x, y)$ and $\psi_{\mathrm{ARM}}(x, y)$ are the complex beam profile functions of the PRC and the arm-cavity modes, representing the electric field amplitude and the phase of the laser beams in a cross sectional plane. These are normalized to make the integral 1 when the two modes are identical.

The plots show that if we want to keep the mode matching above 99\%, the ROC errors of the PR2 and the PR3 have to be less than $5 \mathrm{~mm}$. The $5 \mathrm{~mm}$ ROC error also gives a Gouy-phase deviation of about $4^{\circ}$.

Although keeping the ROC error to be less than $5 \mathrm{~mm}$ out of the $24 \mathrm{~m}$ ROC of the PR3 is not easy to achieve, we can recover the error by changing the distance between the PR2 and the PR3 $\left(l_{\mathrm{p} 2}\right)$. Figure 16 shows how the mode matching and the Gouy-phase error change when $l_{\mathrm{p} 2}$ is changed with ROC errors of $1 \mathrm{~cm}$ added to the PR2 and the PR3. The curves on the plot show the four possible combinations of the signs of the errors on the two mirrors. Since we have to keep the total PRC length constant, $l_{\mathrm{p} 1}$ is changed at every point to compensate for the change in $l_{\mathrm{p} 2}$.

Even with the worst combination of the errors, the mode matching can be recovered by changing $l_{\mathrm{p} 2}$ by roughly the same amount as the ROC error. The one-way Gouy-phase is also recovered to the desired value with the same adjustment of $l_{\mathrm{p} 2}$. When the mirrors are installed, we plan to adjust $l_{\mathrm{p} 2}$, based on the measured value of the ROCs of the fabricated PR2 and PR3. From this observation, the tolerances for the mirror-polishing error of the PR2 and PR3, shown in Table VII, are set to the value by which the suspension systems can be moved without too much difficulty. The effect of the ROC error of the PRM on the mode
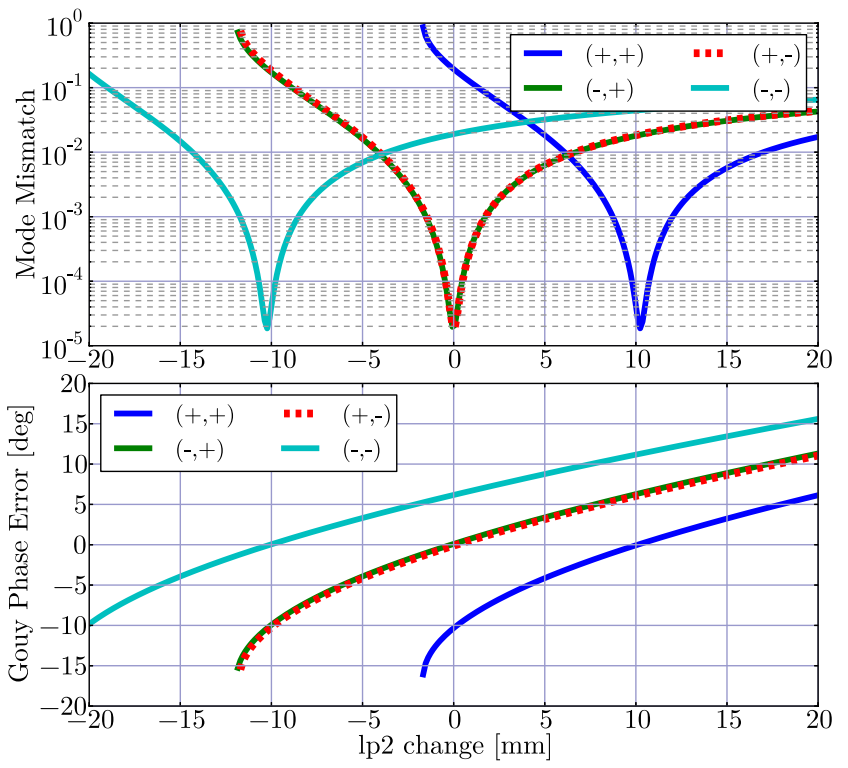

FIG. 16 (color online). The mode mismatch and the Gouyphase error as functions of $l_{\mathrm{p} 2}$. The curves show when ROC errors of $10 \mathrm{~mm}$ are added to the PR2 and the PR3. The signs of the introduced errors are indicated in the legend. 
matching is moderate. We set its error tolerance by requiring that mode matching be greater than $99.99 \%$.

\section{ALIGNMENT SIGNALS}

\section{A. Wave-front sensor shot noise}

With the interferometer parameters selected in the preceding sections, we have to check whether reasonable error signals for the alignment control can be obtained. For the alignment signal extraction, we use the wave-front sensing (WFS) technique [18]. Two quadrant photodetectors (QPDs) are placed at each detection port of the interferometer to receive the output beam at two orthogonal Gouy phases. Signals from each quadrant of the QPDs are demodulated either at the $\mathrm{f} 1$ or the $\mathrm{f} 2$ sideband frequencies. The difference between the demodulated signals from the left quadrants and the right quadrants yields an error signal proportional to the rotations of the mirrors in the horizontal plane (yaw). The difference between the upper and lower quadrants yields an error signal for the bowing rotations of the mirrors (pitch).

In order to compute the WFS signals for a given interferometer configuration, we again use OPTICKLE. By calculating the transfer functions from the rotations of the mirrors to QPD signals at various ports, one can obtain a sensing matrix of dimension (number of signal ports) $\times$ (number of mirrors). Since the sensing matrix is not diagonal in general, we take a linear combination of the QPD signals $q_{n}$, such that $\theta_{m}=\sum_{n} a_{n} q_{n}$, to extract a diagonalized rotation signal $\theta_{m}$ of the $m$ th mirror, where $n$ distinguishes QPDs and $a_{n}$ is the weighting factor. OPTICKLE can also compute the shot noise $s_{n}$ of each QPD output. The total shot noise of each diagonalized signal is calculated by

$$
\theta_{m}^{\text {shot }}=\sqrt{\sum_{n}\left(a_{n} s_{n}\right)^{2}} .
$$

Note that even if the shot noise of each QPD output is small, the diagonalized shot noise can be large, if the mirror rotation signals are nearly degenerated in the QPD signals. Therefore, Eq. (5) can also be used as a figure of merit for good signal separation.

\section{B. Coupling of WFS noises to DARM}

Once the shot noises of the WFS signals are calculated, we now consider their contributions to the bottom-line sensitivity. The rotation $\theta_{m}$ of a mirror is converted to the length change $\delta L_{m}$ of an optical path, coupled with the beam miscentering $d_{m}$ from the rotational center of the mirror,

$$
\delta L_{m}=d_{m} \theta_{m}
$$

For an alignment servo loop with an open-loop gain of $G_{m}(f)$, the WFS shot noise $\theta_{m}^{\text {shot }}$ is converted to the actual rotation $\theta_{m}$ of the mirror by

$$
\theta_{m}(f)=\frac{G_{m}(f)}{1+G_{m}(f)} \theta_{m}^{\text {shot }} .
$$

The transfer function $k_{m}(f)$ from the motion $\delta L_{m}$ of the $m$ th mirror to the DARM signal can be computed with the OPTICKLE model described in Sec. V (see also Ref. [11]). By requiring $k_{m}(f) \delta L_{m}(f)$ to be smaller than the target sensitivity $h(f)$ in the observation frequency band (above $10 \mathrm{~Hz}$ ), we can derive requirements for the WFS shot noise levels as

$$
\theta_{m}^{\text {shot }}<\operatorname{Min}\left[\frac{h(f)}{d_{m} k_{m}(f)} \frac{1+G_{m}(f)}{G_{m}(f)}, f>10 \mathrm{~Hz}\right] .
$$

The beam miscentering $d_{m}$ depends on two factors. One is the static miscentering, which is how well we can adjust a beam-spot position at the center of a mirror. From the experience of the first-generation detectors, we assume the accuracy of this adjustment to be $0.1 \mathrm{~mm}$. Secondly, the alignment fluctuations of the interferometer mirrors cause the beam spots to move around. The conversion coefficient from the rotation angle of a mirror to the beam-spot position changes in the other mirrors can be calculated with OPTICKLE. Assuming rms angular fluctuations of the mirrors to be less than $10^{-8} \mathrm{rad}$, rms beam-spot motions on the mirrors are estimated to be smaller than $0.1 \mathrm{~mm}$. Therefore, we use $d_{m}=0.1 \mathrm{~mm}$ for the test masses in the following calculations. For the other mirrors, we relax the requirement and assume $d_{m}=1 \mathrm{~mm}$.

The assumed angular fluctuation rms of $10^{-8}$ rad comes from the requirement for the beam-jitter coupling, calculated for KAGRA using the method described in Ref. [19]. It has to be ensured by the local damping of the suspension systems in combination with the WFS servo. A detailed analysis of this requires an elaborate suspension model and it will be reported elsewhere. We only make a quick comment here that the rms angular fluctuations of the KAGRA suspensions are mostly determined by rotational resonances below $0.5 \mathrm{~Hz}$. Therefore, it is likely that we can

TABLE VIII. WFS shot-noise requirements and the simulated shot noises. All values are in units of $\mathrm{rad} / \sqrt{\mathrm{Hz}}$.

\begin{tabular}{lcccc}
\hline \hline & \multicolumn{2}{c}{ BRSE } & \multicolumn{2}{c}{ DRSE } \\
\cline { 2 - 5 } & Requirement & Simulated & Requirement & Simulated \\
\hline ETMX & $8.8 \times 10^{-15}$ & $1.9 \times 10^{-14}$ & $9.7 \times 10^{-15}$ & $2.9 \times 10^{-14}$ \\
ETMY & $8.8 \times 10^{-15}$ & $1.9 \times 10^{-14}$ & $9.7 \times 10^{-15}$ & $1.9 \times 10^{-14}$ \\
ITMX & $8.8 \times 10^{-15}$ & $2.8 \times 10^{-14}$ & $9.7 \times 10^{-15}$ & $3.7 \times 10^{-14}$ \\
ITMY & $8.8 \times 10^{-15}$ & $2.8 \times 10^{-14}$ & $9.7 \times 10^{-15}$ & $2.8 \times 10^{-14}$ \\
BS & $9.2 \times 10^{-12}$ & $7.4 \times 10^{-13}$ & $1.5 \times 10^{-11}$ & $3.1 \times 10^{-12}$ \\
PR3 & $3.2 \times 10^{-09}$ & $2.7 \times 10^{-13}$ & $1.4 \times 10^{-09}$ & $1.1 \times 10^{-12}$ \\
PR2 & $3.2 \times 10^{-09}$ & $1.0 \times 10^{-13}$ & $1.4 \times 10^{-09}$ & $3.1 \times 10^{-13}$ \\
PRM & $3.2 \times 10^{-09}$ & $8.9 \times 10^{-14}$ & $1.4 \times 10^{-09}$ & $6.1 \times 10^{-13}$ \\
SR3 & $7.4 \times 10^{-12}$ & $7.7 \times 10^{-12}$ & $1.3 \times 10^{-11}$ & $1.3 \times 10^{-11}$ \\
SR2 & $7.4 \times 10^{-12}$ & $6.6 \times 10^{-11}$ & $1.3 \times 10^{-11}$ & $1.2 \times 10^{-10}$ \\
SRM & $7.4 \times 10^{-12}$ & $1.4 \times 10^{-12}$ & $1.3 \times 10^{-11}$ & $6.8 \times 10^{-12}$ \\
\hline \hline
\end{tabular}



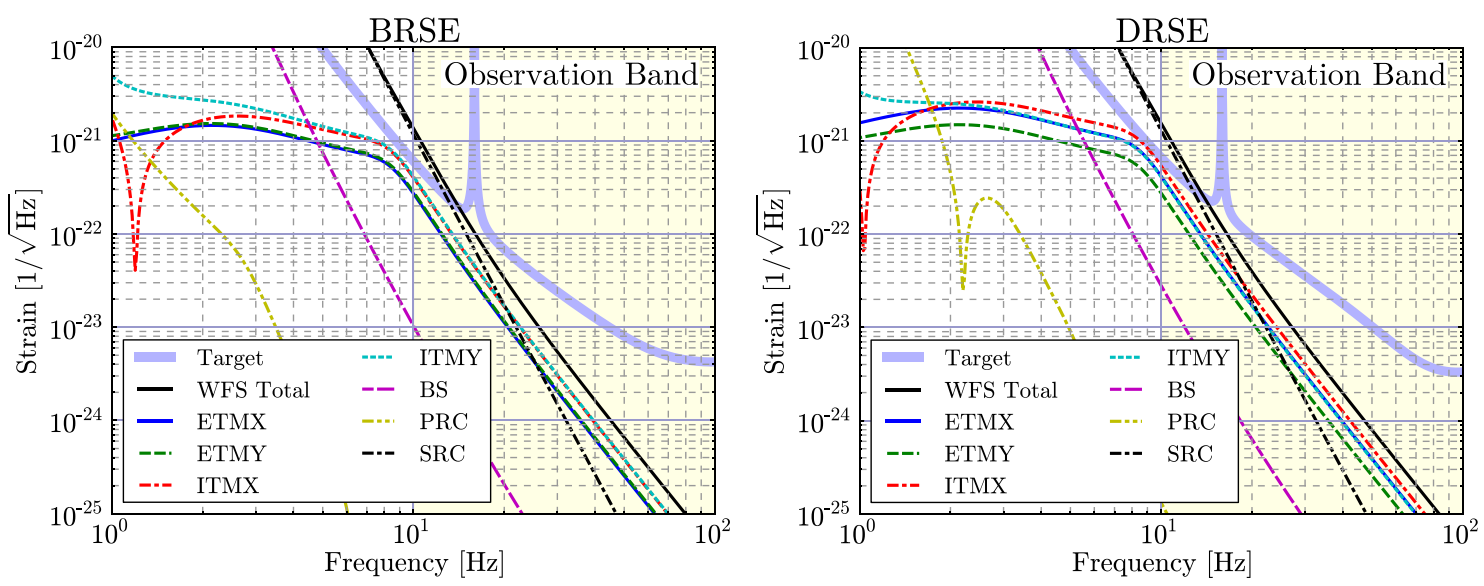

FIG. 17 (color online). Contributions of WFS shot noises to the strain sensitivity. The PRC and the SRC consist of three mirrors each. Therefore, the curves labeled PRC and SRC are the quadratic sums of the noises from the three mirrors.

suppress these resonances by WFS servos with UGFs of $1 \mathrm{~Hz}$ or above, which are assumed in the following analysis.

Table VIII summarizes WFS shot-noise requirements and calculated shot noises for our interferometer configuration. For the calculation of the requirements, we assume the UGFs of $G_{m}(f)$ to be $3 \mathrm{~Hz}$ for the test masses and $1 \mathrm{~Hz}$ for the other mirrors. The shape of $G_{m}(f)$ is $1 / f$ around the UGF and a $1 / f^{4}$ cutoff is added at $10 \mathrm{~Hz}$ for the test masses and at $3 \mathrm{~Hz}$ for the other mirrors. Because the effective resonant frequency of the negative angular optical spring of the arm cavities (Sec. VI A 1) is at about $1 \mathrm{~Hz}$, the WFS servos of the test masses have to suppress this instability. Therefore, the UGF of the test-mass servos is set higher. Each requirement value in the table is computed with Eq. (8) and then divided by $\sqrt{22}$ to take into account the fact that there are incoherent noise contributions from 11 mirrors with two rotational d.o.f. each.

The test masses have 2 to 4 times larger shot noises than the requirements. SR3 and SR2 also do not satisfy the shot-noise requirements. Figure 17 shows the spectral contributions of the WFS shot noises to the strain sensitivity. At the lower edge of the observation band (around $10 \mathrm{~Hz}$ ), the total WFS shot noise exceeds the target sensitivity. In particular, the SRC contribution is large because of the poor shot noise of the SR2 signal. In the actual operation, we may not control the SR2 using WFS or use it only as a DC reference for alignment. Figure 18 shows the WFS shot noises when SR2 is not controlled. In this case, the SRC contribution is reduced significantly. However, the WFS shot noises of the test masses still touch the target sensitivity at $10 \mathrm{~Hz}$.

Although the noise requirements are not strictly satisfied, since the noise excess happens only at the very edge of the observation band, where the sensitivity is not so good in the first place, the impact on the IR is minimum. The calculated IR reductions by the WFS noise are less than $1 \mathrm{Mpc}$ for all the cases. A more serious concern is that in an actual operation the WFS noises may be higher than the estimated shot noises for various technical reasons. We do
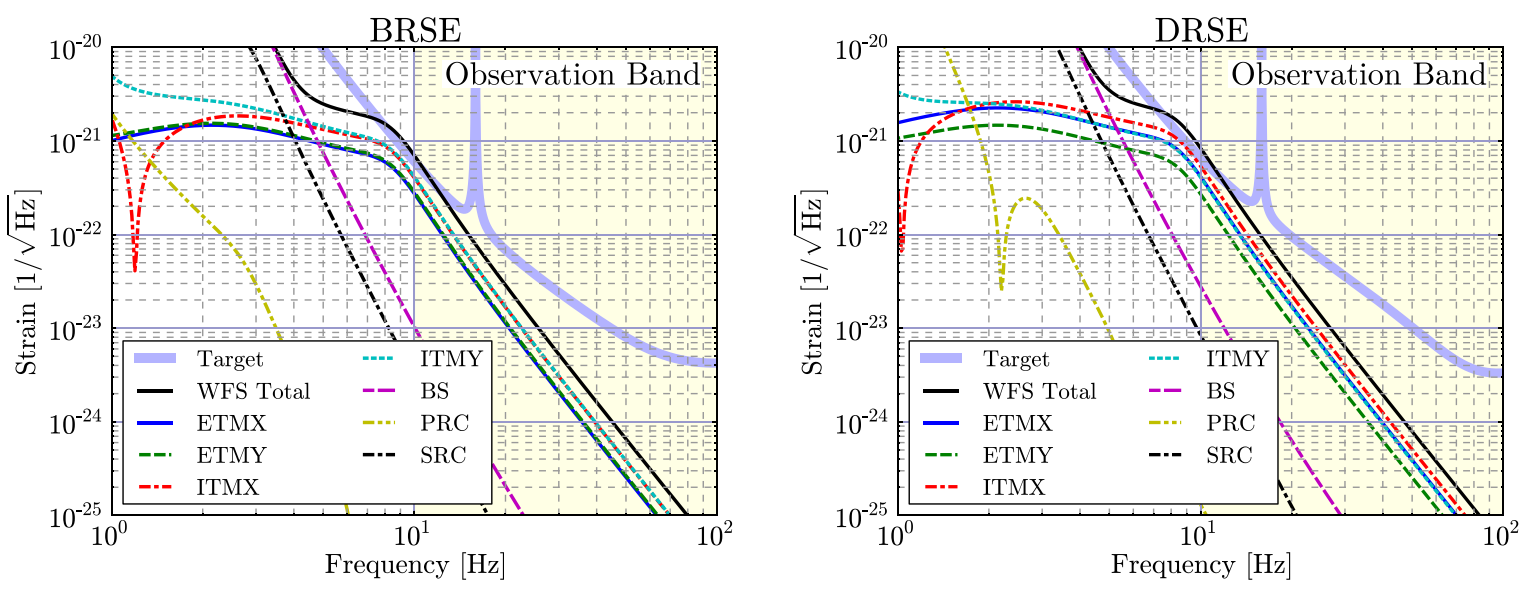

FIG. 18 (color online). Contributions of WFS shot noises to the strain sensitivity when the SR2 is not controlled by WFS. 
not have any safety margin for the test masses to tolerate such a noise increase. In this case, we have to further reduce the beam miscentering, decrease the UGF, or use more aggressive cutoff filters.

\section{CONCLUSION}

We explained the detailed design process of the KAGRA interferometer, starting from classical noises as boundary conditions. Then we optimized the quantum-noise shape using binary inspiral ranges as guidance. Scientific and risk-related reasoning led us to decide to make KAGRA capable of operating in both BRSE and DRSE configurations. We then developed a length-sensing scheme using two sets of RF modulation sidebands. The ROCs of the mirrors were chosen to make the interferometer robust against unwanted higher-order mode resonances. Alignment sensing noise couplings were examined to asses the impact on the target sensitivities.

The most serious concern in the current design is the nearly zero noise margin for the alignment control. Reduction of the alignment control UGFs can reduce the noise couplings significantly. Whether this is possible or not depends on the detailed control design of the suspension systems and their local damping systems. Work on this issue with both computer simulations and experimental verification is on-going.

With the above considerations, the parameters of the KAGRA interferometer are fully determined. Fabrications of the components are now underway and the installations will start in 2014.

\section{ACKNOWLEDGMENTS}

The authors are grateful to Rana Adhikari, Koji Arai, Keita Kawabe, Matt Evans, Lisa Barsotti, and Mike Smith for helpful discussions and advice. This work was supported by the Leading-edge Research Infrastructure Program of Japan. The LIGO Observatories were constructed by the California Institute of Technology and Massachusetts Institute of Technology with funding from the NSF under cooperative agreement PHY-9210038. The LIGO Laboratory operates under cooperative agreement PHY-0107417.
[1] B. Sathyaprakash and B. F. Schutz, Living Rev. Relativity 12 (2009).

[2] J. Hough, Prog. Part. Nucl. Phys. 66, 233 (2011).

[3] KAGRA was formerly called Large-scale Cryogenic Gravitational-wave Telescope (LCGT) [20]. A new nickname KAGRA was given to the detector in 2012.

[4] K. Somiya, Classical Quantum Gravity 29, 124007 (2012).

[5] S. Sato, S. Miyoki, S. Telada, D. Tatsumi, A. Araya, M. Ohashi, Y. Totsuka, M. Fukushima, and M.-K. Fujimoto (LISM Collaboration), Phys. Rev. D 69, 102005 (2004).

[6] R. L. Ward, R. Adhikari, B. Abbott, R. Abbott, D. Barron, R. Bork, T. Fricke, V. Frolov, J. Heefner, A. Ivanov, O. Miyakawa, K. McKenzie, B. Slagmolen, M. Smith, R. Taylor, S. Vass, S. Waldman, and A. Weinstein, Classical Quantum Gravity 25, 114030 (2008).

[7] K. Somiya, Y. Chen, S. Kawamura, and N. Mio, Phys. Rev. D 73, 122005 (2006).

[8] A. Buonanno and Y. Chen, Phys. Rev. D 64, 042006 (2001).
[9] K. Kiuchi, Y. Sekiguchi, M. Shibata, and K. Taniguchi, Phys. Rev. D 80, 064037 (2009).

[10] L. Rezzolla, L. Baiotti, B. Giacomazzo, D. Link, and J. A. Font, Classical Quantum Gravity 27, 114105 (2010).

[11] Y. Aso, K. Somiya, and O. Miyakawa, Classical Quantum Gravity 29, 124008 (2012).

[12] M. Evans, LIGO Document Report No. T070260, 2007.

[13] K. Somiya and O. Miyakawa, Appl. Opt. 49, 4335 (2010).

[14] J. A. Sidles and D. Sigg, Phys. Lett. A 354, 167 (2006).

[15] H. Yamamoto, LIGO Document Report No. T070039, 2007.

[16] Y. Pan, arXiv:gr-qc/0608128.

[17] W. Winkler, K. Danzmann, A. Rüdiger, and R. Schilling, Phys. Rev. A 44, 7022 (1991).

[18] E. Morrison, B. J. Meers, D. I. Robertson, and H. Ward, Appl. Opt. 33, 5041 (1994).

[19] G. Mueller, Opt. Express 13, 7118 (2005).

[20] K. Kuroda, Int. J. Mod. Phys. D 20, 1755 (2011). 